\title{
Pregnant women become insensitive to cold stress
}

\author{
Martin Kammerer $\dagger^{1}$, Diana Adams $\dagger^{2}$, Brida von Castelberg $\dagger^{3}$ and \\ Vivette Glover $\dagger^{* 2}$
}

Address: ${ }^{1} \mathrm{c}$ o University of Zurich, KJPD, Regionalstelle Horgen, Bahnhofstr.6, CH 8810 Horgen, Switzerland, ${ }^{2}$ Wolfson and Weston Research Centre for Family Health, Institute of Reproductive and Developmental Biology, Faculty of Medicine, Imperial College, Hammersmith Campus, Du Cane Road, London, W12 0NN, UK and ${ }^{3}$ Klinik für Gynäkologie und Geburtshilfe, Maternité Inselhof Triemli, Birmensdorferstr.501, CH 8063 Zürich, Switzerland

E-mail: Martin Kammerer - makam1@bluewin.ch; Diana Adams - diana.adams@ic.ac.uk; Brida von Castelberg - makam1@bluewin.ch;

Vivette Glover* - v.glover@ic.ac.uk

*Corresponding author †Equal contributors

Published: 19 November 2002

BMC Pregnancy and Childbirth 2002, 2:8

This article is available from: http://www.biomedcentral.com/ |47|-2393/2/8

(C) 2002 Kammerer et al; licensee BioMed Central Ltd. This is an Open Access article: verbatim copying and redistribution of this article are permitted in all media for any purpose, provided this notice is preserved along with the article's original URL.
Received: 26 July 2002

Accepted: 19 November 2002

\begin{abstract}
Background: The function of the hypothalamic-pituitary-adrenal (HPA) axis is known to be altered during pregnancy, but it has not been tested with a natural stressor.

Methods: A group of pregnant women $(n=10)$ were tested towards the end of pregnancy (mean $36.8 \pm 2.5$ weeks gestation) and about 8 weeks postpartum (mean $7.8 \pm 1.5$ weeks), together with a matched control group, with a one minute cold hand stressor test. Saliva samples were collected before and 10 and 20 minutes after the test, and stored for later radioimmunoassay of cortisol.

Results: The control group showed a highly significant response to the test. The pregnant group showed no response, and the postpartum group a variable but non significant one

Conclusions: This shows that the HPA axis becomes hypofunctional to a natural stressor at the end of pregnancy. It is suggested that one possible evolutionary function for this is to protect the fetus from the stress responses of the mother.
\end{abstract}

\section{Background}

It is established that the function of the hypothalamic-pituitary-adrenal (HPA) axis is altered during human pregnancy, although much remains to be understood concerning this. CRH is released from the placenta into the maternal blood as pregnancy progresses, and plasma levels of cortisol also increase [5]. The HPA axis becomes hypofunctional at the end of pregnancy in response to a CRH challenge [18]. It is also insensitive to feedback inhibition by dexamethasone, and this has been shown to persist into the postpartum period [17].
There is clearcut evidence from animal studies that if the mother is stressed during pregnancy that this can have long term effects on the behaviour of the offspring $[19,23]$. There is also evidence that this can be mediated via her HPA axis. In non human primates the effects are abolished by adrenalectomy, and mimicked by administration of ACTH [20]. There is evidence in humans that prenatal stress and anxiety can have effects on the pregnancy and they have been linked with pre-term delivery [13]. Maternal anxiety during pregnancy can also have long term effects on the behaviour of the child $[10,16]$. 
There is a good correlation between maternal and fetal plasma cortisol levels $[8,9]$, and it is quite possible that raised cortisol in the pregnant mother has a direct effect on the development of the fetus and the fetal brain [9]. Different individual women have a wide range of cortisol levels during pregnancy $[8,9]$. It is thus important to understand more about what causes some women to have higher cortisol levels than others, and the nature of the cortisol response to stress during human pregnancy.

In this study we have used the cold hand test to study the saliva cortisol responses of women at the end of pregancy and in the postpartum period, compared with controls. This is a test, which has been widely used to test HPA responsivity in non-pregnant subjects, and causes an increase in saliva cortisol [e.g. [2]].

\section{Methods}

Ten healthy pregnant women, with no medical disorder, were recruited from the antenatal clinic of the Maternité Inselhof Triemli Hospital in Zurich at $36.8 \pm 2.5$ weeks antenatally (mean \pm SD) and followed postnatally at $7.8 \pm$ 1.5 weeks. Eight of the 10 were still breast-feeding at that stage. Exclusion criteria were known abnormality of the fetus, artificial insemination, and inadequate German or English. Ten non-pregnant healthy controls matched for age and parity were recruited in the Zurich school of midwifery. Psychiatric disorder was excluded by using the Structured Clinical Interview for DSMIV diagnoses SCID [22].

The test was started at the same time of day for each woman, pregnant and postpartum (mean for the group 12 noon \pm 1.2 hours). All controls were tested in the same time period. The subjects put their non dominant hand into ice cold water for one minute. Saliva cortisol was taken just before and 10 and 20 minutes after the cold test was carried out.

Saliva was collected with salivettes and removed from the dental rolls by centrifugation. Cortisol levels were assayed using a standard solid phase radioimmunoassay, adapted for use with saliva as recommended by the manufacturer (Euro DPC, Gwynedd, UK). The lower limit of sensitivity for the saliva assay is $1.5 \mathrm{nmol} / \mathrm{L}$.

\section{Results}

Fig 1 shows that the controls showed the expected highly significant increase in saliva cortisol 20 minutes after immersing the hand in ice cold water $(\mathrm{p}<0.001)$. This response was generally absent in the group of pregnant women who showed no significant mean increase, despite a small increase in some individuals. The effect was quite variable in the postpartum women. Some showed a large increase, others not and the increase for the group as a whole was not significant. The results were similar after excluding the two who were not breast-feeding.

Fig 2 shows the differences between the three groups. Fig 2a shows that the baselines were very different, the pregnant group having the highest values, the postpartum intermediate and the controls had the lowest values. Fig $2 b$ shows the reverse pattern for the responsivity. There was a significant difference in the degree of response between the pregnant and control groups $(\mathrm{p}<0.05)$ but not between the other groups.

\section{Discussion}

This study has shown quite clearly that the cortisol response to the cold hand stress test was considerably dampened in women at the end of pregnancy. This confirms and extends previous studies on the function of the HPA axis during pregnancy, although none has looked directly at the cortisol response to a natural stressor rather than a pharmacological challenge. Schulte and colleagues showed that women at one week prior to their delivery date were generally insensitive to the stimulation of ACTH and cortisol by a bolus of CRH [18]. However, interestingly, one woman did experience emotional and physical stress during the test, and she reacted with a sharp rise in cortisol. The present results may also explain those of Gitau et al [9] who found that pregnant women (22 to 35 weeks gestation) showed no significant cortisol response to the experience of fetal blood transfusion, a procedure which one would expect to be stressful and which caused a marked rise in noradrenaline [7].

As in previous studies, we have found here that the baseline levels in the pregnant women were considerably higher than controls [1,3]. It has been known for some time that the plasma concentration of both total cortisol and free cortisol increase during pregnancy and peak at the end of the third trimester [1]. It is also well established that CRH, which is undetectable in the plasma outside pregnancy, rises exponentially in the maternal bloodstream during gestation, due to release from the placenta. It is plausible that the high cortisol and CRH act by feedback mechanisms to blunt the HPA responsivity to stress.

The question of the extent to which the CRH drives the rise in cortisol is of interest. Goland et al 1994 [12] showed a positive correlation between plasma ACTH and CRH concentrations and levels of urinary free cortisol and suggested that the placental CRH was a driving force behind the gestational hypercortisolism. Magiakou et al 1996 [14] have shown that ACTH and cortisol maintain a circadian rhythm during pregnancy whereas CRH does not. They also showed a strong correlation between ACTH and cortisol, both in magnitude and pulses, but none between CRH and ACTH in late pregnancy. They suggest that 


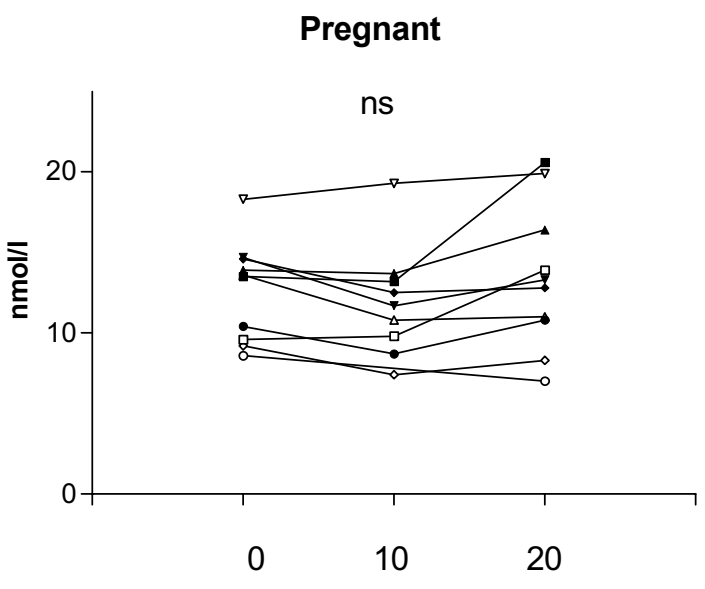

Post partum
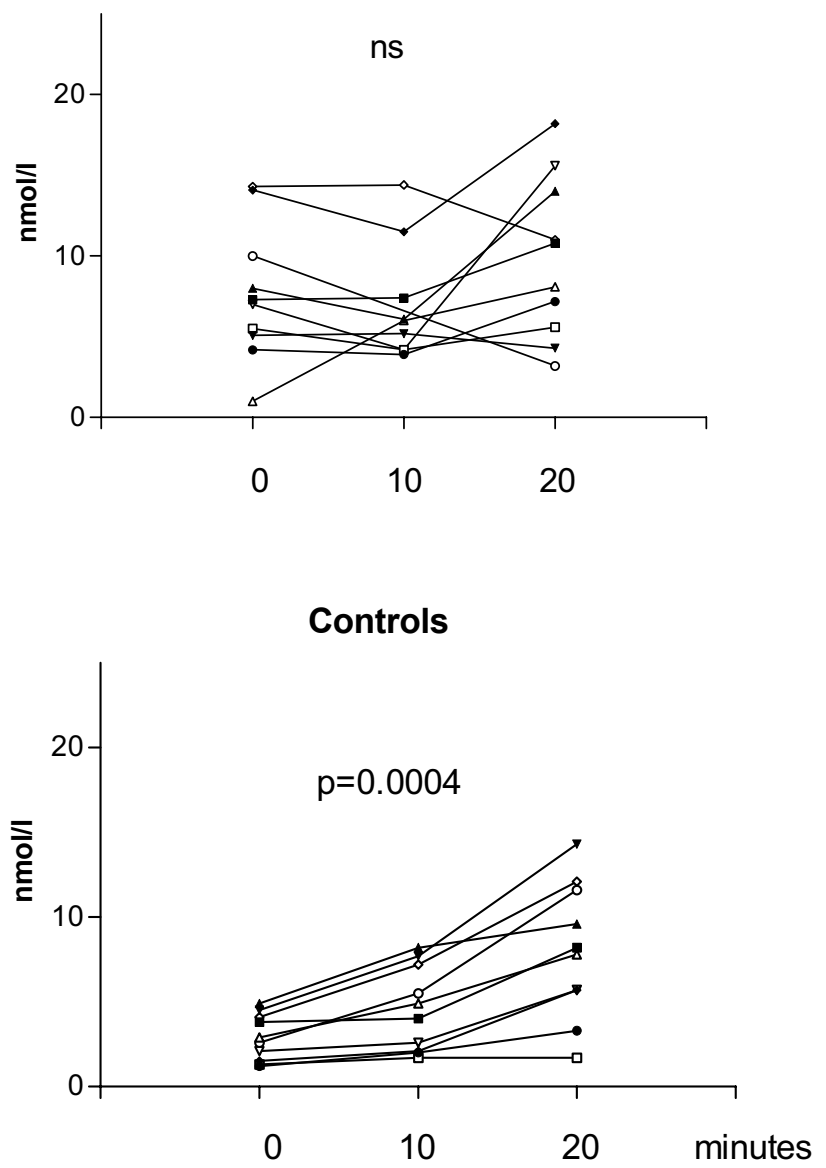

Figure I

Saliva cortisol before and 10 and 20 minutes after immersion of hand in ice cold water. Analysis by paired $t$ test comparing values at 0 and 20 minutes Pregnant n.s; postpartum ns; controls $\mathrm{p}=0.0004$.
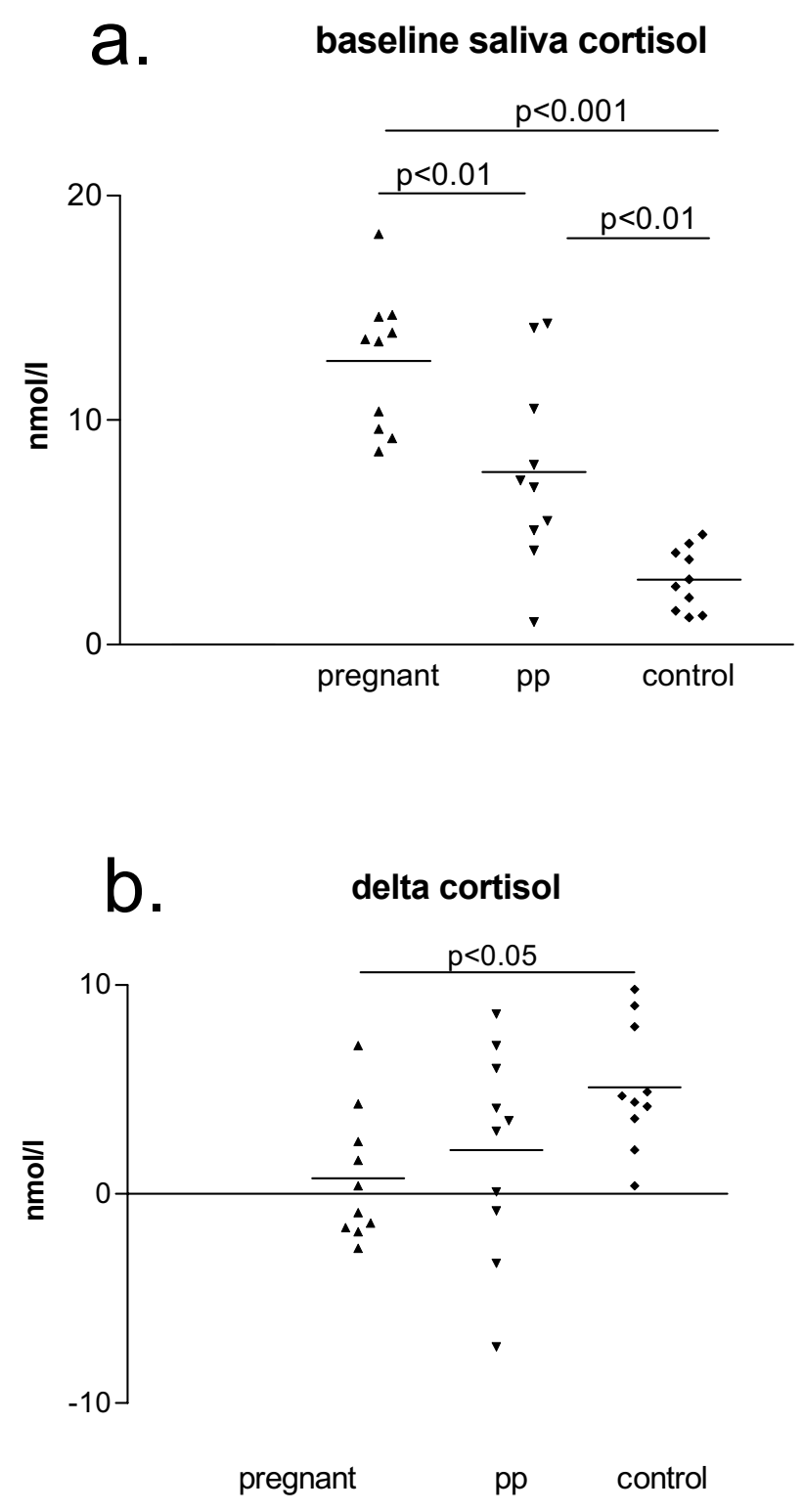

Figure 2

a. Comparison of basal ( 0 minutes) saliva cortisol levels in the 3 groups. One way Anova $p<0.0001$. Pregnant vs postpartum $p<0.01$; pregnant $v$ control $p<0.001$; postpartum versus control $p<0.01$, all after Bonferroni corrections. b. Comparison of delta values (20-0 minutes) in the 3 groups. One Way Anova $p=0.03$. Pregnant $v$ controls $p<0.05$. Others ns after Bonferroni corrections. All significance values are from a 2 tailed analysis.

although the overall hypercortisolaemia of pregnancy may be driven by placental $\mathrm{CRH}$, there must be another major ACTH secretagogue, and that the most likely candidate is arginine vasopressin (AVP). This is secreted into the portal system by parvocellular neurones of the paraventricular nucleus of the hypothalamus, in a pulsatile 
fashion with a circadian increase in amplitude. It has been suggested that this AVP stimulated system enables the pregnant woman to continue to respond to stress even if the response to $\mathrm{CRH}$ is desensitised. However the results presented here suggest that the whole physiological system becomes desensitised.

The postpartum women were intermediate in all the parameters studied between the controls and pregnant groups. Even though almost 8 weeks postpartum, their baseline cortisol values had not returned to control levels. They showed a variable response to the cold stress test, not significant in itself but not significantly different from either the pregnant or the control groups. It will be of interest to determine, in future studies, whether their responses return to normal after lactation is finished. Owens et al (1987) [17] found that the dexamethasone test was abnormal in the first two to three postpartum weeks but had returned to normal by 35 days, or about 6 weeks postpartum. However Magiakou et al, 1996 [15] showed that there was a blunted ACTH response to a bolus of oCRH at both 3 and 6 weeks postpartum, which only returned to normal by 12 weeks. It is plausible that this adjustment, which is similar to that seen after treatment of Cushing's disease, contributes to the vulnerability to emotional lability and depression which is common in the postpartum period.

It is also of interest that basal maternal cortisol is still high in many of the postpartum women. It is known that cortisol is present in breast milk and is inversely related to immunoglobulin A [11], and this raises the possibility that there could be direct effects on the baby.

The current study raises several questions. It is important to determine when exactly in pregnancy the cortisol response starts to become dampened, and when postpartum it becomes fully functional again. Does it become insensitive to psychological or cognitive as well as physical stress? Singh et al 1999 have shown that in general men who responded highly to a physical stressor also responded more to a psychological stressor [21]. The fact that the CRH challenge test, the dexamethasone challenge test and baseline cortisol values are all altered, suggests that it is not solely the response to cold that is altered in pregnancy and the postpartum period, but the general function of the HPA axis.

It is intriguing to ask what the evolutionary function of the development of this insensitivity may be. The most plausible explanation of the strong correlation between maternal and fetal cortisol levels $[8,9]$ is direct transfer across the placenta. Cortisol crosses membranes easily, and as the maternal fetal ratio is over 10/1 this is compatible with considerable metababolism of cortisol as it crosses the placenta. If cortisol from the mother can affect fetal brain development [8], and possibly time of parturition, it may be important to buffer the fetus from these effects, especially in later pregnancy.

It is of note that within the pregnant group, while most showed no response to the cold stressor, four did show some increase in saliva cortisol. This complements the finding of Schulte et al (1990) [18], who showed that one individual did show a response to the CRH challenge. Depressed, stressed or anxious women generally have raised cortisol levels [4], and depression is common during pregnancy [6]. It will be of interest to determine whether women who are anxious or depressed while pregnant show higher baseline cortisol levels and greater reactivity than others.

\section{Conclusions}

More needs to be understood concerning the functioning of the HPA axis during human pregnancy and in the postpartum period, and how it is affected at different gestational ages in women who are stressed, anxious or depressed. To do this we need a simple test that is acceptable to pregnant women, who may not want to be exposed to a drug challenge. The cold hand stress test appears suitable for this function.

\section{Competing interests}

None declared.

\section{Author's contributions}

Author BvC was in clinical charge of the patients in the antenatal clinic. Author MK collected all the samples. Author DA assayed them. Author VG conceived the study. All authors participated in its detailed design and read and approved the final manuscript.

\section{Acknowledgements}

MK is grateful to the Swiss Federal Office for Sciences for support (BBW, Nr. 97.0034). VG and DA were supported by the Henry Smith Charity.

\section{References}

I. Abou-Samra AB, Pugeat M, Dechaud $H$, Nachury L, Bouchareb B, Feure-Montange $M$, Tourniare J: Increased plasma concentrations of $\mathrm{N}$-terminal $\beta$-lipotropin and unbound cortisol during pregnancy. Clin Endocrinol 1984, 20:22I-228

2. al'Absi M, Petersen KL, Wittmers LE: Adrenocortical and hemodynamic predictors of pain perception in men and women. Pain 2002, 96: 197-204

3. Allolio B, Hoffmann J, Linton EA, Winkelmann W, Kusche M, Schulte HM: Diurnal salivary cortisol patterns during pregnancy and after delivery: relationship to plasma corticotrophin-releasing-hormone Clin Endocrinol (Oxf) 1990, 33:279-89

4. Checkley S: The neuroendocrinology of depression and chronic stress. Br Med Bull 1996, 52:597-617

5. Chrousos GP, Torpy DJ, Gold PW: Interactions between the hypothalamic-pituitary-adrenal axis and the female reproductive system:clinical implications. Ann Intern Med 1998, I 29:229240 
6. Evans J, Heron J, Francomb H, Oke S, Golding J: Cohort study of depressed mood during pregnancy and after child birth. $B M J$ 200I, 23:257-260

7. Giannakoulopoulos X, Teixeira J, Fisk N, Glover V: Human fetal and maternal noradrenaline responses to invasive procedures. Pediatr Res 1999, 45:494-499

8. Gitau R, Cameron A, Fisk NM, Glover V: Fetal exposure to maternal cortisol. Lancet 1998, 352:707-8

9. Gitau R, Fisk NM, Teixeira JM, Cameron A, Glover V: Fetal hypothalamic-pituitary-adrenol stress responses to invasive procedures are independent of maternal responses. J Clin Endocrinol Metab 200I, 86:104-9

10. Glover $\mathrm{V}, \mathrm{O}$ 'Connor TG: Effects of antenatal stress and anxiety: Implications for development and psychiatry. Brit J Psychiat 2002, 180:389-391

II. Groer MW, Humenick S, Hill PD: Characterisations and psychoneuroimmunologic implications of secretory immunoglobulin A and cortisol in preterm and term breast milk. Perinat Neonatal Nurs 1994, 7:42-51

12. Goland RS, Jozak S, Conwell I: Placental corticotropin-releasing hormone and the hypercortisolism of pregnancy. Am J Obstet Gynecol 1994, I71:|287-I29|

13. Hedegaard M, Henriksen TB, Sabroe S, Secher NJ: Psychological distress in pregnancy and preterm delivery. BMJ 1993, 307:234-239

14. Magiakou MA, Mastorakos G, Rabin D, Margioris AN, Dubbert B, Calogero AE, Tsigos C, Munson PJ, Chrousos GP: The maternal hypothalamic-pituitary-adrenal axis in the third trimester of human pregnancy. Clin Endocrinol (Oxf) 1996, 44:419-428

15. Magiakou MA, Mastorakos G, Rabin D, Dubbert B, Gold PW, Chrousos GP: Hypothalamic corticortopin-releasing hormone suppression during the postpartum period: implications for the increase in psychiatric manifestations at this time. J Clin Endocrinol Metab 1996, 81:1912-7

16. O'Connor TG, Heron J, Golding J, Beveridge M, Glover V: Maternal Antenatal Anxiety and Children's Behavioural/Emotional Problems at 4 Years. Brit J Psychiat 2002, 180:502-508

17. Owens PC, Smith R, Brinsmead MW, Hall C, Rowley M, Hurt D, Lovelock M, Chan EC, Cubis J, Lewin T: Postnatal disappearance of the pregnancy-associated reduced sensitivity of plasma cortisol to feedback inhibition. Life Sci 1987, 41:1745-50

18. Schulte HM, Weisner D, Allolio B: The corticotrophin releasing hormone test in late pregnancy: lack of adrenocorticotrophin and cortisol response. Clin Endocrinol (Oxf) 1990, 33:99106

19. Schneider ML, Roughton EC, Koehler AJ, Lubach GR: Growth and development following prenatal stress exposure in primates: an examination of ontogenetic vulnerability. Child Dev 1999 70:263-74

20. Schneider M, Moore CF: Effect of prenatal stress on development: A nonhuman primate model. In: Minnesota Symposium on Child Psychology (Edited by: Nelson C) New Jersey: Erlbaum 2000, 201243

21. Singh A, Petrides JS, Gold PW, Chrousos GP, Deuster PA: Differential hypothalamic-pituitary-adrenal axis reactivity to psychological and physical stress / Clin Endocrinol Metab 1999, 84:1944-8

22. Spitzer R, Williams JBW, Gibbon M, First MB: The structured clinical interview for DSM-IIIR (SCID) Arch Gen Psychiat 1992, 49:624-629

23. Weinstock M: Alterations induced by gestational stress in brain morphology and behaviour of the offspring. Prog Neurobiol 200I, 65:427-5I

\section{Pre-publication history}

The pre-publication history for this paper can be accessed here:

http://www.biomedcentral.com/1471-2393/2/8/prepub

\footnotetext{
Publish with BioMed Central and every scientist can read your work free of charge

"BioMedcentral will be the most significant development for disseminating the results of biomedical research in our lifetime."

Paul Nurse, Director-General, Imperial Cancer Research Fund

Publish with $\mathrm{BMC}$ and your research papers will be:

- available free of charge to the entire biomedical community

- peer reviewed and published immediately upon acceptance

- cited in PubMed and archived on PubMed Central

- yours - you keep the copyright

Submit your manuscript here:

BioMedcentral.com

http://www.biomedcentral.com/manuscript/
} 